\title{
Haptic shape perception from force and position signals varies with exploratory movement direction and the exploring finger
}

\author{
KnUt Drewing and LuKas KaIm \\ Giessen University, Giessen, Germany
}

\begin{abstract}
We investigated how exploratory movement influences signal integration in active touch. Participants judged the amplitude of a bump specified by redundant signals: When a finger slides across a bump, the finger's position follows the bump's geometry (position signal); simultaneously, it is exposed to patterns of forces depending on the gradient of the bump (force signal). We varied amplitudes specified by force signals independently of amplitudes specified by position signals. Amplitude judgment was a weighted linear function of the amplitudes specified by both signals, under different exploratory conditions. The force signal's contribution to the judgment was higher when the participants explored with the index finger, as opposed to the thumb, and when they explored along a tangential axis, as opposed to a radial one (pivot $\cong$ shoulder joint). Furthermore, for tangential, as compared with radial, axis exploration, amplitude judgments were larger (and more accurate), and amplitude discrimination was better. We attribute these exploration-induced differences to biases in estimating bump amplitude from force signals. Given the choice, the participants preferred tangential explorations with the index finger-a behavior that resulted in good discrimination performance. A role for an active explorer, as well as biases that depend on exploration, should be taken into account when signal integration models are extended to active touch.
\end{abstract}

Haptic perception is inherently active (Gibson, 1962). It depends on exploratory movements that generate the sensory signals. How redundant signals from active touch are integrated into a coherent percept has recently been described by established integration models (Drewing \& Ernst, 2006; Drewing, Wiecki, \& Ernst, 2008; cf. Ernst \& Banks, 2002). There also exist data that suggest that exploratory behavior influences signal integration. Here, we asked whether influences from exploratory behavior can also be described by the established models. We studied how manipulations of exploratory movement (direction in horizontal plane; using the index finger vs. the thumb) influence the integration of force and position signals into haptically perceived shape, and we asked whether participants prefer exploratory behavior that optimizes shape perception.

Signal integration has been studied extensively for cases in which observers have not much control over how they obtain information (e.g., for visual depth signals, such as texture and disparity). Jacobs (2002) suggested that such integration takes into account all signals available for a property. Signal-specific estimates, $\hat{s}_{i}$, for that property are derived from each signal, $s_{i}$. Then all the estimates combine into a percept, $\hat{s}$, by weighted averaging:

$$
\hat{s}=\sum_{i} w_{i} \hat{s}_{i} \text { with } \sum_{i} w_{i}=1 ; w_{i} \in[0,1] .
$$

Weighted averaging describes the percept of a property well when stimuli with signals that conflict in their information on this property are created (Backus, Banks, van Ee, \& Crowell, 1999; Young, Landy, \& Maloney, 1993). Averaging different estimates has been further predicted to increase the percept's reliability, $R_{\hat{s}}\left(=1 / \sigma_{\hat{S}}^{2}\right.$, inverse of the percept's variance; Landy, Maloney, Johnston, \& Young, 1995). This prediction was confirmed by lower discrimination thresholds for a property in multisignal, as compared with single-signal, situations (Perotti, Todd, Lappin, \& Phillips, 1998). In addition, it has been suggested that the signal estimates' weights in the percept shift with the estimates' reliabilities (reliability-dependent weighting). Indeed, adding external noise to the display of one signal lowered the reliability of that signal's estimate and the estimate's weight in the percept (Backus et al., 1999; Young et al., 1993). Although most authors agree that the weights depend on reliabilities, there is some controversy over the situations in which weights are set "optimally" (Rosas, Wagemans, Ernst, \& Wichmann, 2005). Optimal integration, as defined by the maximum-likelihood estimation model, produces the percept with highest reliability, $R_{\hat{S}}$. Optimal weights, $w_{i}$, are proportional to the normalized signal estimates' reliabilities, $R_{i}$ :

$$
w_{i}=\frac{R_{i}}{\sum_{j=1 \ldots, i, \ldots N} R_{j}} \text { with } R_{\hat{s}}=\sum_{i} R_{j} .
$$

Both optimal (Alais \& Burr, 2004; Ernst \& Banks, 2002; Hillis, Watt, Landy, \& Banks, 2004) and suboptimal (Gep-

K. Drewing, knut.drewing@psychol.uni-giessen.de 
shtein, Burge, Ernst, \& Banks, 2005; Rosas et al., 2005) reliability-dependent weighting have been observed.

Also, during active touch, observers combine redundant signals (Srinivasan \& LaMotte, 1995; Voisin, Lamarre, \& Chapman, 2002). Drewing et al. (2008; Drewing \& Ernst, 2006) investigated the integration of force and position signals in haptic shape perception (Robles-De-La-Torre $\&$ Hayward, 2001). When a finger slides across a bump on a surface, the finger follows the geometry of the bump (position signal to shape; finger lowers and rises). Simultaneously, the finger is exposed to patterns of forces that depend on the gradient of the surface (reaction forces from the object that are normal to the surface). Changes in force direction during exploration perturb the finger in its trajectory and create a force signal to shape. These force and position signals are normally highly correlated and, thus, are redundant signals to shape. Using a force feedback device, the authors created virtual 3-D arches in which the force signal specified one curvature of the arch, while the position signal specified a different curvature. It was found that both signals contributed to judged curvature; the judgment was a weighted average of the two specified curvatures. Moreover, the signals' weights shifted with variables that affected the signals' reliabilities. For example, when exploring soft objects, as compared with hard objects, the finger follows a more variable path across the object's surface, presumably lowering the reliability of the position signal estimate for curvature. Along with this, the position signal weight decreased (Drewing et al., 2008). In conclusion, the established assumptions - weighted averaging and reliability-dependent weighting - described the integration of the two signals into a shape percept quite well. At the same time, signal weights considerably differed between individuals and were correlated with exploratory velocity and force (Drewing \& Ernst, 2006). These correlations might also indicate reliability-dependent weighting. Exploration might have influenced the signals' reliabilities and, thus, indirectly affected the weights.

Here, we investigated experimentally how exploratory movement influences the integration of force and position signals into the percept of a virtual bump. Kaim and Drewing (2007) manipulated exploratory velocity and force. The present study manipulated the finger used in exploration (index finger or thumb) and the direction of movement in the horizontal plane (Figure 1B): Movement proceeded along one of the two oblique axes that were about radial and tangential to a circle described by a vertical axis rotation around the shoulder joint, and it started at one of the two possible startpoints of each axis. We argue that exploratory direction influences the force signal estimate's reliability, whereas the finger used influences both the force and position signal estimates' reliabilities. The manner of exploration should influence signal estimates, because it determines which and how many individual receptor cells sense a signal. Note that receptors are unequally distributed in the skin. Exploratory direction within the horizontal plane determines which side of the finger (left, right, tip of the finger, etc.) approaches the bump on the plane first. This finger's side then determines which individual receptors in the finger sense the force signal, because the force signal has a lateral effect on the finger. But the finger's side does not affect the sensing of the position signal, which has a vertical effect on finger position. In contrast, different fingers contain different individual receptors, and thus, the finger used in exploration affects the sensing of both signals. From differences in sensing, we expect differences in the estimates' reliabilities. However, we do not predict the direction of differences.

In two experiments, we tested whether the effects of our exploratory conditions on the integration of force and position signals are sufficiently described by reliability shifts, weighted averaging, and reliability-dependent weighting of signal estimates. The experiments were designed simultaneously and complement one another. In Experiment 1, we used a quick measure of felt bump amplitude (subsequent adjustment of the amplitude of a visual bump), which allowed us to systematically vary force and position signals for amplitude. We tested whether both force and position signals would contribute to perceived amplitude and whether their integration would follow a weightedaveraging scheme for each exploratory condition. We further tested whether exploratory conditions would affect the weighting of signal estimates, which would be indicated by effects on force signal contributions to the percept that were mirrored by opposite effects on position signal contributions (because weights sum to 1). In Experiment 2, we used a two-interval forced choice task (participants compared a standard bump with discrepant force and position signals to bumps with consistent signals), which allowed us to measure the reliability of perceived amplitude (via just-noticeable differences [JNDs]) and signal weights for a subset of conditions. Experiment 2 was designed to directly test whether effects of exploratory direction follow from reliability-dependent weighting: In this case, force signal estimate weight and the percept's reliability should covary across exploratory directions, because exploratory direction affects the force, but not the position, signal estimate's reliability. If the force signal estimate's weight depends on the estimates' reliabilities, it will shift with the force signal estimate's reliability. The reliability of the percept shifts similarly, because it originates from the single estimate's reliabilities. Finally, by extending the idea of optimal integration to optimal exploration, we tested whether, in spontaneous exploration, participants prefer movements that maximize the percept's reliability (see Lederman \& Klatzky, 1987, for corresponding findings regarding disparate movement schemes).

\section{EXPERIMENT 1}

We created haptic stimuli by using a PHANToM force feedback device. The device is attached to a finger via a thimble. It simulates objects by monitoring 3-D finger position and by applying an appropriate reaction force. If the finger "indents" a virtual object, a reaction force drives the finger out of the object. Usually, reaction force linearly increases with indentation depth, and force direction is normal to the surface geometry. We changed this rendering method. First, with the standard method, 
A

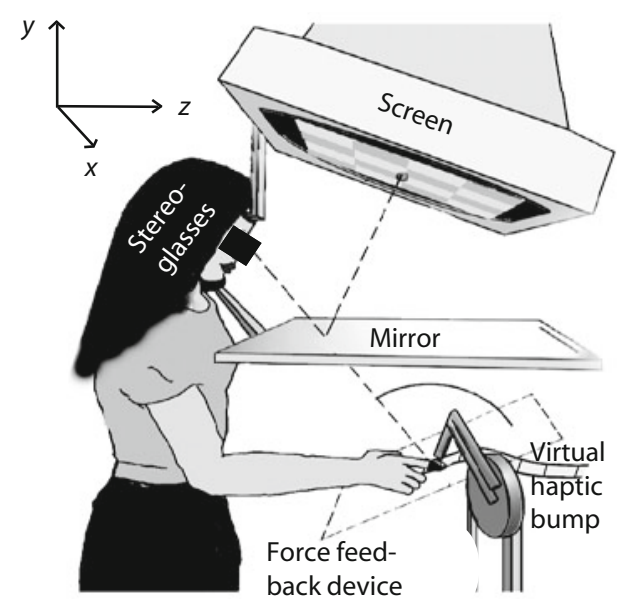

B

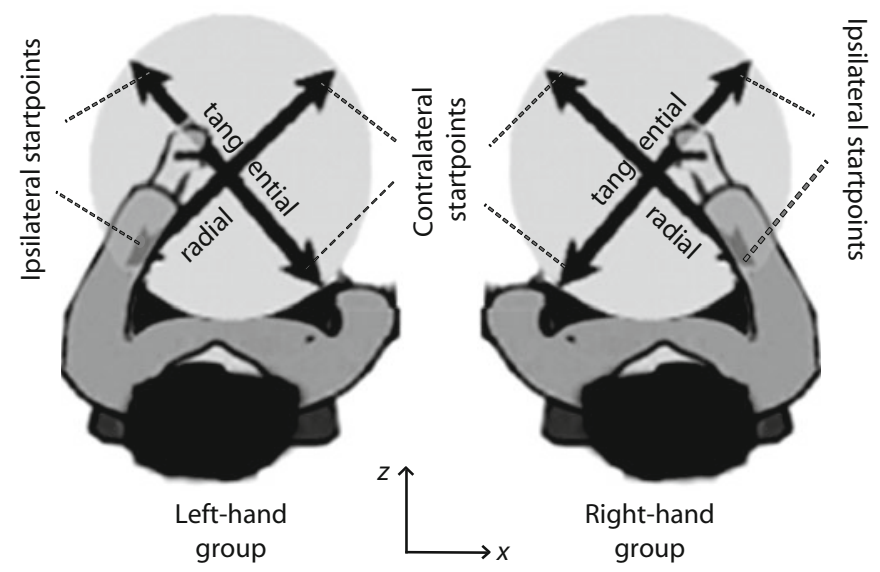

C

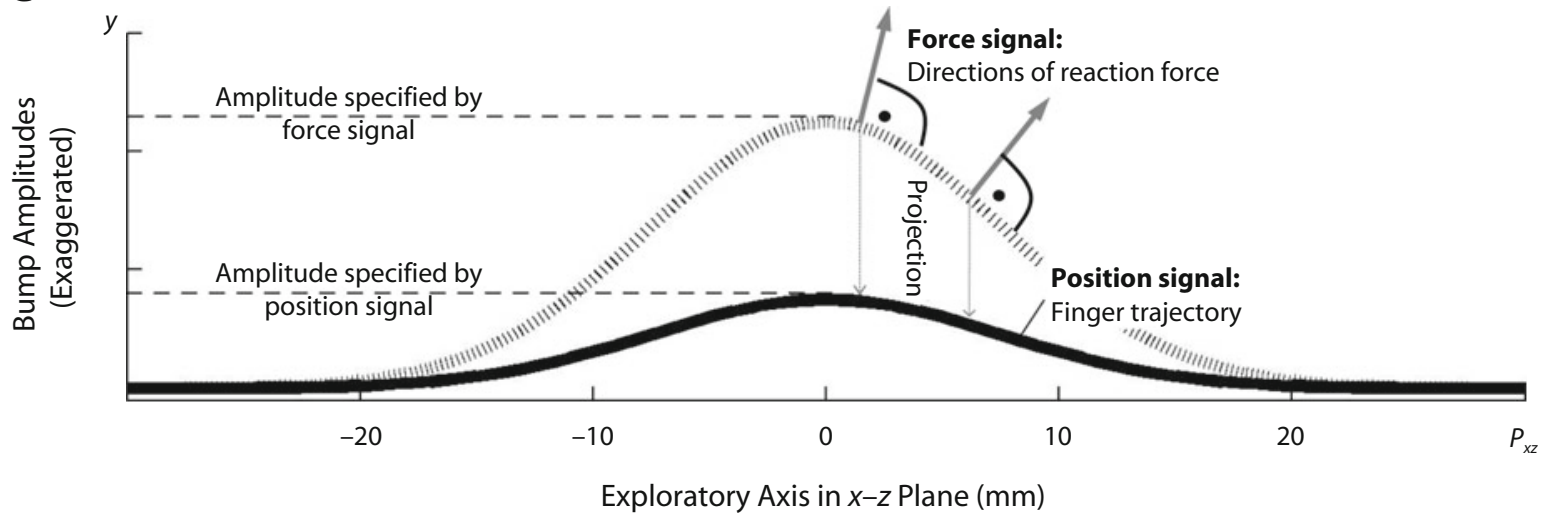

Figure 1. (A) Visuohaptic setup. Note that the participants were sitting. (B) Exploratory directions and nomenclature in reference to the shoulder joint (@ pivot). (C) Force and position signals (amplitudes exaggerated). When exploring natural bumps, the finger follows the geometry of the shape (position signal) and experiences reaction forces that are normal to the geometry (force signal); in our stimuli, force directions of a Gaussian-shaped bump with one amplitude (dashed line) were orthogonally projected on the geometry of a bump with another amplitude (solid line).

the finger's force deforms the object's surface and, correspondingly, influences the shape of the finger trajectory across the surface (Drewing et al., 2008). We improved the method so that the finger followed a trajectory that hardly depended on finger force or velocity anymore. Basically, the finger was maintained on the target trajectory by including finger force and future target positions of the finger into the calculation of reaction force (details below). Second, a surface rendered in standard manner provides force and position signals that are both consistent with the rendered shape. Here, we separately manipulated the force and position signals. The geometry of the bump determined the shape of the finger trajectory (position signal) via the magnitude of the reaction force. The directions of the reaction forces were taken from a bump with a different amplitude and were orthogonally projected onto the geometry. This specified force signals that were different from the ones dictated by geometry (Figure 1C). When the finger stroked across the bump, the force signal determined horizontal forces acting on the finger in the direction or counterdirection of the stroke, whereas the position signal determined the amplitude of vertical finger displacement. Thus, force and position signals were independent. Note also that the PHANToM's thimble creates a permanent contact between finger and environment, which might or might not act as an additional but constant signal to shape.

We used nine standard stimuli (Gaussian-shaped bumps). While independently varying force and position signals for bump amplitudes of 3.0, 4.5, and $6.0 \mathrm{~mm}$, we constructed nine pairings of force and position signals; three of the pairings had consistent signals, six did not. The bumps were explored with either the thumb or the index finger in the four different exploratory directions in the horizontal plane (Figure 1B). Participants explored with a prescribed finger velocity $(15 \mathrm{~cm} / \mathrm{sec})$ and force $(2 \mathrm{~N})$ guided by abstract visual feedback on their current finger velocity and force. We measured felt amplitude from 
the participants' adjustments of the amplitude of a visual bump presented subsequently. After the main experiment, the participants participated in a free exploration block, in which they were asked to choose the exploring finger (thumb or index finger) and the exploratory direction that would yield the "best perceptual performance." The best participant was rewarded (€20).

\section{Method}

Participants. Eight students (naive as to the purpose of the study; right-handed; 4 females; ages, 19-43 years, $M=25$ ) without any sensory or motor impairments participated for course credit.

Apparatus. The participants sat in front of a visuohaptic setup (see Figure 1A) comprising a PHANToM 1.5A $(1000 \mathrm{~Hz}, 0.03-\mathrm{mm}$ resolution, force in three translatory directions) and a 22-in. monitor $(120 \mathrm{~Hz})$. A finger was connected to the device via a thimble-like holder, which allowed for free movements in a $38 \times 27 \times 20 \mathrm{~cm}^{3}$ workspace. The participants' heads were stabilized by a chinrest. They looked through stereoglasses (Crystaleyes) and, via a mirror, at the monitor (40-cm viewing distance). The mirror prevented the participants from seeing their hand and enabled spatial alignment of the 3-D visual and haptic displays. The devices were connected to a PC. Custom software controlled the experiment, collected responses, and recorded finger positions and reaction forces (from PHANToM, every $10 \mathrm{msec}$ ).

Stimuli. The PHANToM applies reaction forces, $\vec{F}_{P}$, as a function of the 3-D finger position $P$. With standard rendering, force magnitude linearly increases with the indentation depth of the finger into a virtual object $\left(i_{P}\right.$; here, distance of finger to virtual surface along $y$-axis, according to Figure 1), and force direction is normal to the object's surface $\left(\vec{n}_{P}\right.$, normal vector; here, at surface point with finger's $x$ - and $z$-coordinates; $D$, spring constant):

$$
\vec{F}_{P}=\vec{n}_{P} \cdot\left|\vec{F}_{P}\right|, \quad\left|\vec{F}_{P}\right|=D \cdot i_{P} .
$$

We improved this method. The spring constant $D$ was replaced by the variable $K$ in order to keep object indentation constant under differing finger forces. The variable $K$ was defined such that for the target indentation $I$ (set to $1 \mathrm{~mm}$ ), the magnitudes of finger force and reaction force were (approximately) equal. Vertical finger force was estimated from the device's reaction forces in $y$-direction $F_{y}(j)$ in the previous device cycles $j=1 \ldots n(\sim$ previous $300 \mathrm{msec})$ :

$$
K=\frac{\frac{1}{n} \sum_{j=1 \ldots n} F_{y}(j)}{I} .
$$

The calculation of reaction force magnitude was further improved by a factor $T$ (having effects similar to a damping factor) and an anticipation factor $Q$ (Equation 5 replaced Equation 3, right part). Both factors reduce short-term deviations of the finger from a target trajectory:

$$
\begin{aligned}
& \left|\vec{F}_{P}\right|=K \cdot i_{P y} Q \cdot T, \\
& \text { with } T=\left(1-v_{y} \cdot E\right)^{2} \\
& \text { and } Q=1+\frac{g\left(P_{x z_{-} \text {pred }}\right)-g\left(P_{x z}\right)}{P_{x z_{-} \text {pred }}-P_{x z}} .
\end{aligned}
$$

The factor $T$ implements a percentage correction of the reaction force that counteracts vertical finger vibration $\left(v_{y}\right.$ : vertical finger velocity, $E=1 \mathrm{msec} / \mathrm{mm}$ ). The anticipation factor $Q$ equals 1 plus the slope of the target trajectory for the next millisecond $\left[P_{x z}\right.$, current 1-D finger position along the exploration axis; $P_{x z_{-} \text {pred }}$, position predicted for the next millisecond; $g\left(P_{x z}\right)$, shape's height]. Thus, the factor $Q$ increases the reaction force when the finger should rise in the next millisecond and vice versa. There are other options to damp and to control trajectories anticipatorily, but our method efficiently maintained the finger on the trajectories for the given shapes and exploratory constraints (cf. the Results section). Shapes were Gaussian bumps:

$$
g\left(P_{x z}\right)=A * e^{-\frac{P_{x z}^{2}}{2 \sigma^{2}}} .
$$

The bump's amplitude $A$ varied (3.0-6.0 $\mathrm{mm} ; \sigma=7.5 \mathrm{~mm})$. We dissociated force and position signal amplitudes (Figure 1C). One function, $g_{P}\left(P_{x z}\right)$ with one amplitude $A_{P}$, defined the shape of the target trajectory via reaction force magnitude (Equation 5; position signal). Another function, $g_{F}\left(P_{x z}\right)$ with amplitude $A_{F}$, defined the forces' directions [normals of $g_{F}\left(P_{x z}\right)$; Equation 3 left; force signal]. Bumps were presented on an otherwise planar area of 320$\mathrm{mm}$ length (exploration axis) and $15-\mathrm{mm}$ width. The bump's peak amplitude was located $+50 \mathrm{~mm}$ or $-50 \mathrm{~mm}$ from the center of the exploration axis; strokes started $-150 \mathrm{~mm}$ from the center.

Design and Procedure. The experimental design consisted of five within-participants variables: finger (index finger or thumb), axis (radial or tangential), startpoint (ipsi- or contralateral to exploring hand), force signal $(3.0,4.5$, or $6.0 \mathrm{~mm})$, and position signal $(3.0,4.5$, or $6.0 \mathrm{~mm})$. The hand used in exploration (left or right) was balanced across participants. Axis and startpoint varied in the horizontal plane directly in front of the participant (Figure 1B); the exploratory axes were the two diagonals in the workspace $\left( \pm 45^{\circ}\right.$ rotation of the $x$-axis around the $y$-axis). Exploratory startpoints were at either side of the axes. For each combination of exploratory conditions (finger $\times$ startpoint $\times$ axis), we displayed nine standard bumps for all combinations of position and force signal amplitudes (12 repetitions/condition).

Each trial started with a visual representation of the upcoming exploratory axis and startpoint. The participants initiated the trial with a buttonpress at the startpoint location and then made a single stroke across the stimulus. During the stroke, a vertical line that moved along the exploratory axis (moving again and again from start- to endpoint) indicated the prescribed finger velocity $(15 \mathrm{~cm} / \mathrm{sec})$. A stationary horizontal line indicated prescribed force $(2 \mathrm{~N})$. The participants monitored their current velocity and force by further feedback lines (not displayed in bump area). A vertical line displayed the current 1-D finger position on the axis; a horizontal line moved up and down with exerted force. Otherwise, the screen was black. After the stroke, the participants adjusted the amplitude of the drawing of a Gaussian-shaped bump ( $\sigma=7.5 \mathrm{~mm}$; initial amplitude, $0-90 \mathrm{~mm}$; rotated into viewer plane, centered) to the amplitude previously felt (using two buttons). Buttonpresses were done with the PHANToM.

The experiment consisted of four sessions ( $\sim 70 \mathrm{~min}$ each). Sessions conducted with the index finger alternated with sessions conducted with the thumb. Each session consisted of a block of trials in which the participants were trained in achieving the prescribed force and velocity and of an experimental block ( 6 repetitions/standard for each axis $\times$ startpoint combination; $6 \times 9 \times 4=216$ trials; order randomized). Trials were repeated later in the block when actual velocity or force values deviated too much from the prescribed ones. At the end of the final session, an extra free exploration block was conducted (144 trials): The participants chose the exploring finger (thumb or index finger) that they thought would yield the "best perceptual performance." On each trial, they chose one of the four combinations of startpoint and axis.

Data analysis. The primary variable was the visually adjusted amplitudes. These scores were averaged for each individual (12 trials/condition). For each exploratory condition and each individual, we then calculated a multiple linear regression of adjusted amplitudes $A_{V}$ on force and position signal amplitudes $A_{F}$ and $A_{P}$ (nine stimuli/regression):

$$
A_{V}=c_{P} A_{P}+c_{F} A_{F}+b
$$

The regression coefficients $c_{P}$ and $c_{F}$ are estimates of the position and force signal contributions to adjusted amplitude. Pilot data sug- 
gested that adjusted amplitudes are not equal to the haptic percept $\hat{s}$ but, rather, to a linear function of it ( $v$ : scaling):

$$
A_{V}=v \hat{s}+\text { const. }
$$

In the pilot study, there was a large range of amplitudes (consistent signals, $A_{P}=A_{F}=1,2, \ldots 7 \mathrm{~mm}, N=4$ ). The data revealed good fits $\left(r^{2}: 9-1.0\right)$ for linear regressions of adjusted amplitudes on signal amplitudes. But the slopes varied considerably between individuals (.2-.8). This suggests that the scaling of perceived into adjusted amplitude depends on individual preferences and that the measures above, $c_{P}$ and $c_{F}$, contain a subjective scaling component. In Experiment 1, we hence derived the force signal weight $w_{F}^{e l}$ that allowed for scaling:

$$
w_{F}^{e 1}=\frac{c_{F}}{c_{P}+c_{F}} .
$$

The weight $w_{F}^{e 1}$ is an estimate of the estimate's weight $w_{F}$ in Equation $1\left(=1-w_{p}\right)$, given that (1) Equation 8 holds, (2) only force and position signal estimates contribute to the percept, and (3) signal estimates equal the signals $\left(\hat{s}_{i}=A_{i}\right)$. Inserting the latter two assumptions into Equation 1,

$$
\rightarrow \hat{s}=w_{F} A_{F}+\left(1-w_{F}\right) A_{P},
$$

insert in Equation 8,

$\rightarrow A_{V}=v w_{F} \cdot A_{F}+v\left(1-w_{F}\right) \cdot A_{P}+$ const,

compare with Equation 7, and solve for $w_{F}$,

$$
\rightarrow c_{F}=v w_{F}, c_{P} v\left(1-w_{F}\right), w_{F}=\frac{c_{F}}{c_{P}+c_{F}}=w_{F}^{e 1} .
$$

Note that if either of the above assumptions is violated, $w_{F}^{e 1}$ will not be an estimate of the weight $w_{F}$ in Equation 1 but an estimate of a more complicated term. This term is described by Equation 11A for the case in which, in addition to force and position signals, another constant signal contributes to the percept. The term is described by Equation $11 \mathrm{~B}$ for the case in which signal estimates do not equal the signals, but the mapping $\hat{s}_{i}=m_{i} A_{i}$ includes a factor $m_{i} \neq 1$ :

$$
\begin{gathered}
\frac{w_{F}^{e}=w_{F}}{\left(w_{F}+w_{p}\right)} \\
\frac{w_{F}^{e}=m_{F} w_{F}}{\left(m_{F} w_{F}+m_{P} w_{p}\right)} .
\end{gathered}
$$

In the interest of brevity, we will not provide further detail. But note that Equations 11A and 11B also hold true for empirical signal weights in Experiment $2\left(w_{F}^{e 2}\right)$ and in Landy et al. (1995).

We further analyzed finger position and reaction force data in order to check whether actual vertical finger displacements matched with the defined bump amplitudes and whether actual finger forces and velocities were similar between conditions.

\section{Results}

Adjusted amplitudes. Adjusted amplitudes (see Figure 2) increased with increases in both position and force signal amplitude. The increase of adjusted amplitude with force signal amplitude was larger when participants explored along the tangential axis than when they explored along the radial axis and when they explored with the index finger, as compared with the thumb. The average magnitude of adjusted amplitudes depended on exploratory direction (radial-ipsi/contra, 3.9/3.9 mm; tangential-ipsi/ contra, 4.6/4.4 mm). Adjusted amplitudes were entered into an ANOVA (variables: finger, axis, startpoint, force signal, and position signal). The effects of force signal $[F(2,14)=68.8, p<.001$; if necessary, $p$ values were cor- rected according to Geisser \& Greenhouse, 1958], position signal $[F(2,14)=28.3, p<.001]$, and axis $[F(1,7)=$ $49.6, p<.001]$ were statistically significant, as were the axis $\times$ startpoint $[F(1,7)=6.9, p<.05]$, finger $\times$ force signal $[F(2,14)=5.3, p<.05]$, and axis $\times$ force signal $[F(2,14)=9.5, p<.01]$ interactions.

Regression analyses. Regression analyses (see Figures $3 \mathrm{~A}-3 \mathrm{D}$ ) demonstrated that a linear combination of force and position signal amplitudes described the adjusted amplitudes well. Contributions of the position signal and regression intercepts were similar in all the exploratory conditions; the force signal contribution and the derived force signal weight were larger for tangential than for radial explorations and for index finger than for thumb explorations. Statistically, the linear regression model explained most of the variance in the majority of individual data sets (median $r^{2}=.76$; first quartile, .62; third quartile, .89). Regression parameters $\left(c_{F}, c_{P}, b\right)$ and force signal weights $w_{F}^{e 1}$ were analyzed by four ANOVAs (variables: startpoint, axis, and finger). For force signal contributions $\left(c_{F}\right)$ and force signal weights, there were significant main effects of finger $\left[c_{F}, F(1,7)=7.52, p<\right.$ $\left..05 ; w_{F}^{e 1}, F(1,7)=5.9, p<.05\right]$ and of axis $\left[c_{F}, F(1,7)=\right.$ $\left.12.52, p<.01 ; w_{F}^{e 1}, F(1,7)=5.6, p<.05\right]$. No other effect in either of the analyses was significant (all $p \mathrm{~s}>.2$ ).

Manipulation check. We calculated the actual amplitude of vertical finger displacement during the exploration of each bump. Across the 72 within-participants conditions, averaged actual amplitudes ranged from $98 \%$ to $103 \%$ of the targeted amplitudes as defined by the position signal. Averaged standard deviations between trials did not exceed $0.2 \mathrm{~mm}$ in either condition. These deviations are small and will be ignored in further analyses. We further calculated exploratory finger forces and velocities (just in front of each bump). Individual means were entered into ANOVAs (variables: axis, startpoint, and finger). Velocity was significantly direction dependent [tangential-ipsi/contra, 14.3/14.5 cm/sec; radial, $12.7 / 12.9 \mathrm{~cm} / \mathrm{sec}$; axis, $F(1,7)=17.1, p<.01$; axis $\times$ startpoint, $F(1,7)=5.6, p<.05]$. Exploration was more forceful along the radial than along the tangential axis $[1.97$ vs. $1.94 \mathrm{~N} ; F(1,7)=7.5, p<.05]$. These potential confounds will be discussed below. Other effects were not significant $(p>.10)$.

Exploratory preferences. All the participants chose to conduct the free exploration block using the index finger, not the thumb (see Figure 3E). We tested preferences displayed in trial-by-trial choices separately for each of the six possible pairings within the four exploratory directions (for instance, tangential-ipsi vs. radial-contra): A preference for one among two directions is indicated by choosing that direction with a relative frequency greater than random $50 \%$. We tested these relative frequencies against 50\% using binomial tests. Overall, the participants significantly preferred the two tangential over the two radial directions (four tests, $p \mathrm{~s}<.001$ ), with a slight preference for contralateral startpoints within the radial axis $(p<.01)$; startpoints did not differ within the tangential axis $(p>.5)$. In individual analyses, 7 out of 8 participants significantly preferred one direction over all others 
Tangential Exploration

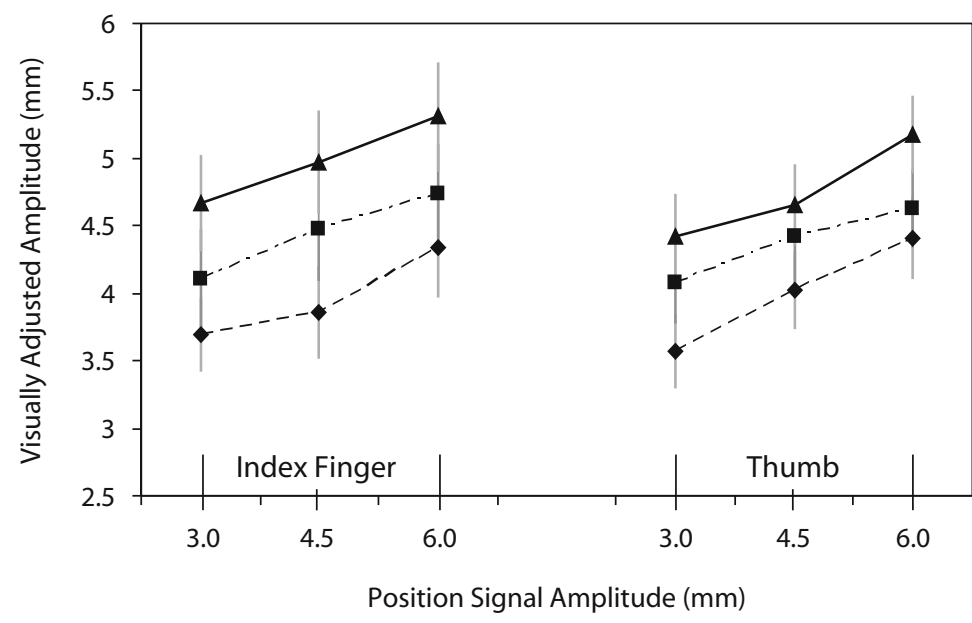

Radial Exploration
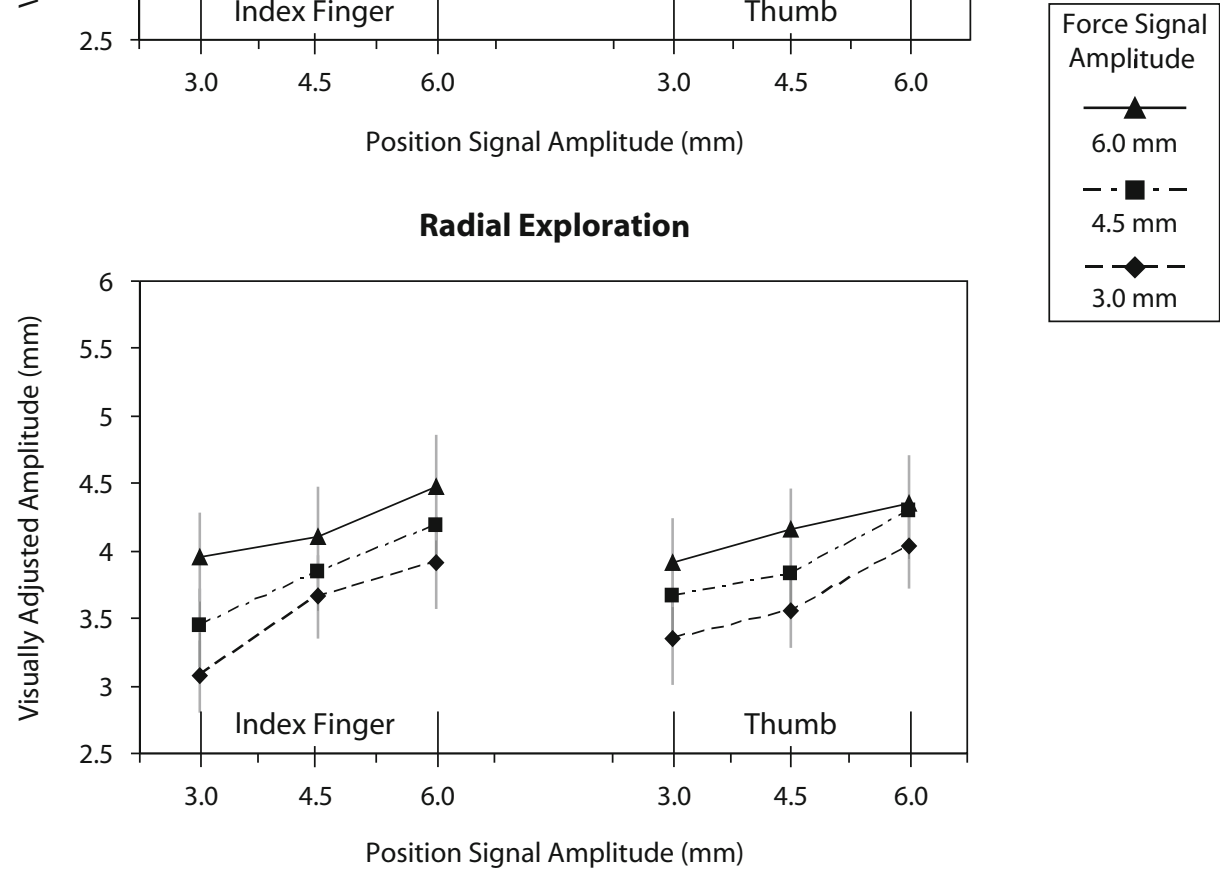

Figure 2. Experiment 1: Visually adjusted amplitudes and standard errors (8 participants) as a function of force signal, position signal, finger, and axis (collapsed across startpoint).

$(p<.01$ in each of the six individual tests); in six cases, this was a direction along the tangential axis.

\section{Discussion}

In Experiment 1, we investigated the integration of force and position signals to perceived bump amplitude as a function of exploratory direction and the finger used. The two signals were systematically varied. The participants adjusted the amplitude of a visual bump to felt amplitude. Adjusted amplitudes increased with the amplitudes specified by force and by position signals, and linear regressions described well the relation between adjusted and signal amplitudes for each exploratory condition. We conclude that, as was predicted, the integration of estimates from force and position signals into perceived amplitude follows a weightedaveraging scheme under each exploratory condition.

The overall magnitude of adjustments differed across exploratory conditions, suggesting that manner of exploration affected ( $=$ differentially biased) perceived ampli- tude. We had, in particular, expected exploratory effects on the weighting of force and position signal estimates in the percept. These would have been indicated by effects on force signal contributions to adjusted amplitude that are mirrored by opposite effects on position signal contributions. Force signal contributions were larger when the participants explored along the tangential axis than when they explored along the radial axis and when they used the index finger rather than the thumb. Position signal contributions, however, did not vary at all. This indicates that the position signal estimate's weight did not shift and, thus, also that the force signal estimate's weight did not shift, because weights sum to 1 . This conclusion is in contrast to our prediction and already argues against an explanation of exploratory effects by reliability-dependent estimate weighting. Experiment 2 tested whether reliabilities actually differ between conditions, as we presumed.

How can we, then, explain the exploratory effects on the force signal contribution? The force signal contribution to 


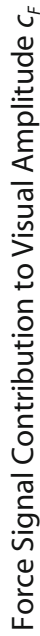

A

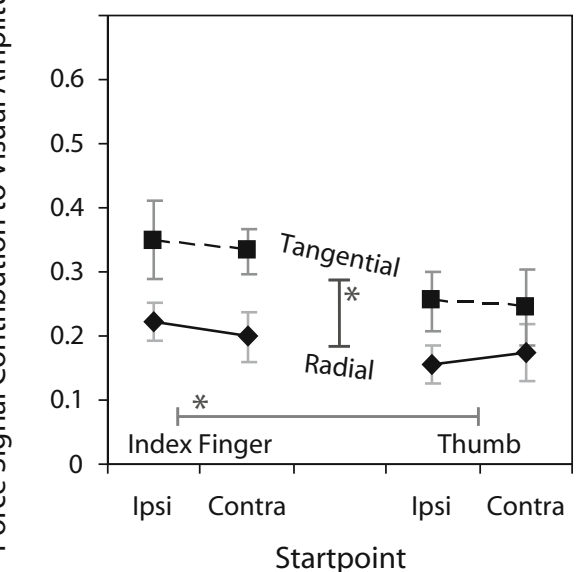

C

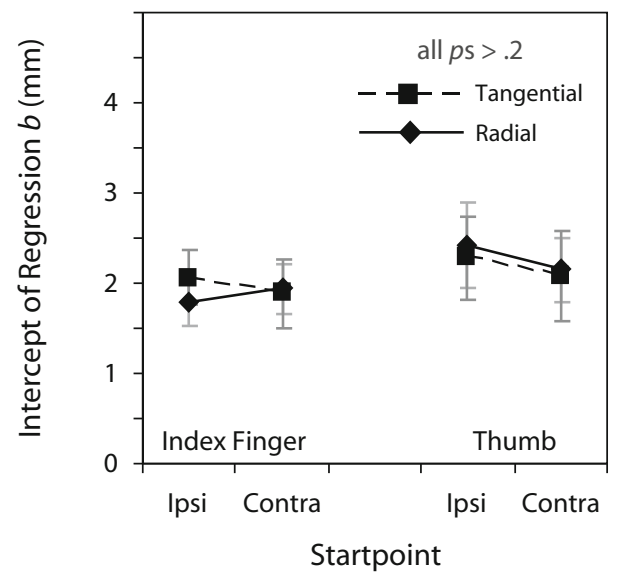

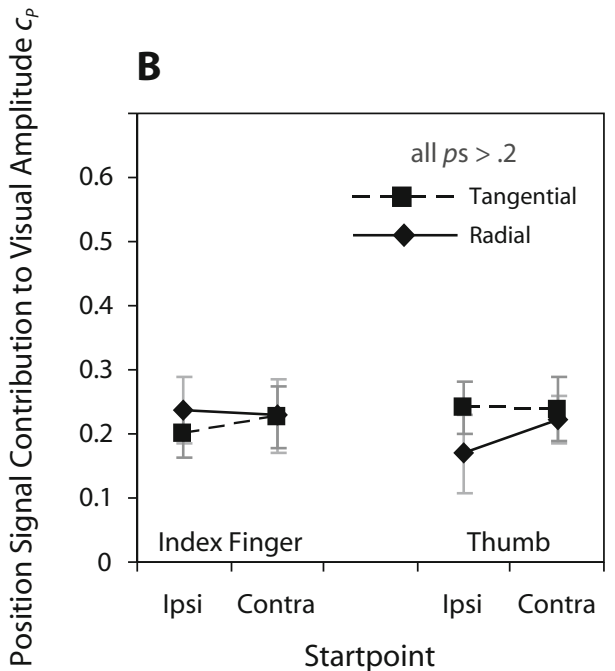

D

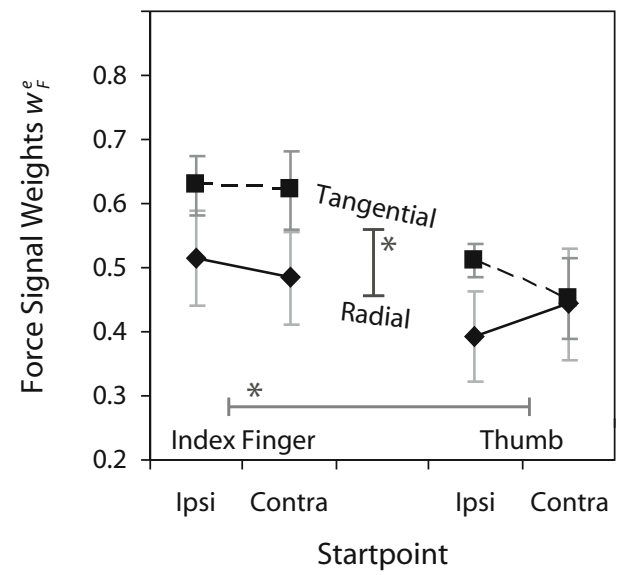

$\mathbf{E}$

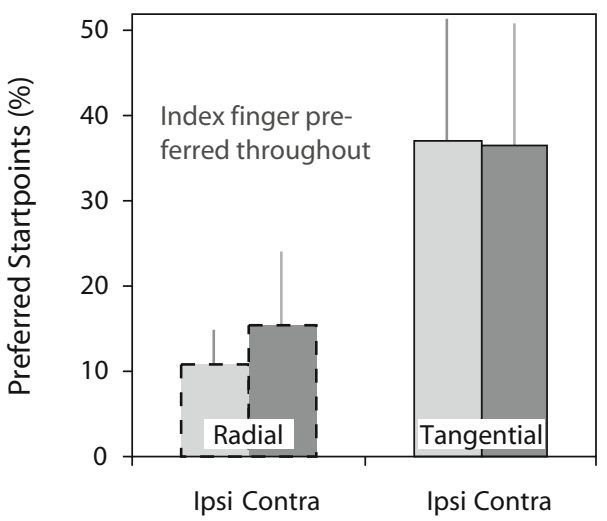

Startpoint

Figure 3. Experiment 1. (A-D) Results from linear regressions of adjusted amplitudes on amplitudes specified by force and position signals as a function of axis, startpoint, and finger: (A) regression coefficient $c_{F}$ (force); (B) regression coefficient $c_{P}$ (position); (C) intercept $b$; (D) force signal weight. (E) Percentages and standard errors (8 participants) of participants' choices of startpoint and axis in free exploration. 
adjusted amplitudes depends on the estimate's weight $w_{F}$ in the percept, as well as on the mapping of the force signal amplitude $A_{F}$ on the amplitude estimate $\hat{s}_{F}$. Given that estimate weights did not shift, we conclude that the mapping $\hat{s}_{F}=m_{F} A_{F}$ included a factor $m_{F} \neq 1$ (i.e., a bias), which was larger in tangential than in radial exploration and in index finger than in thumb exploration. That is, estimates derived from the same force signal amplitudes had larger values in the corresponding conditions. These biases explain exploratory effects in the force signal contribution, in the derived force signal weight (via violation of assumptions, Equation 11B), and they predict corresponding overall perceptual biases, which we observed at least for tangential, as compared with radial, exploration.

Note also that there was a "remainder" in describing adjusted amplitudes from force and position signals, a positive regression intercept. The intercept might be an artifact of the visual adjustment method. Or, the PHANToM's thimble might have acted as an additional constant signal to amplitude, and the intercept reflects the corresponding estimate. This, however, does not weaken our conclusion. If the intercept reflected another estimate, the lack of effect in intercept additionally argues against shifts in the estimates' weights. Another objection against our conclusion could be that tangential explorations were performed more quickly and with less force than radial ones. This confound, however, cannot explain the larger force signal contributions in tangential than in radial exploration. When force and velocity were manipulated experimentally (Kaim \& Drewing, 2007), both faster and less forceful exploration decreased the force signal contribution, rather than increasing it.

Finally, we investigated exploratory preferences, when participants were rewarded for good perceptual performance. Being free to choose, all the participants explored with the index finger, not with the thumb. In trial-by-trial choices, they preferred tangential to radial explorations. Most individuals ( 7 out of 8 ) also displayed a clear preference for a single exploratory direction; 6 preferred the tangential axis in combination with one of the two startpoints. Importantly, preferred exploratory conditions are those with the most accurate visual adjustment of bump amplitude (see Figure 2). Biomechanical factors appear unlikely to explain the preferences: Trial repetition due to imprecise movement was similar for tangential (10\%) and radial (11\%) exploration. In Experiment 2, we investigated whether the chosen axis actually would produce better performance in a discrimination task.

\section{EXPERIMENT 2}

Experiment 2 varied exploratory direction (axis, startpoint) only. It was designed to complement Experiment 1 by measures of the percept's reliability. It, thus, allowed us to relate exploratory preferences and exploratory effects on signal processing with reliability shifts (in particular, with shifts in force signal estimate reliability; see the introduction). We presented three standard bumps (position signal amplitude $A_{P} /$ force signal amplitude $A_{F}[\mathrm{~mm} / \mathrm{mm}]$ : $3 / 6,4.5 / 4.5,6 / 3$ ) and several comparison bumps with consistent force and position signals. On each trial, the participants explored a standard and a comparison with one stroke that crossed the two bumps and afterward decided which bump had felt higher. Using the method of constant stimuli, we measured JNDs and points of subjective equality (PSEs; the amplitude of the comparison that was perceived as the same amplitude as the standard). The JNDs were used to estimate reliability. From the PSEs of the two standards with discrepant signals $\left(A_{P} / A_{F}: 3 / 6\right.$, $6 / 3$ ), we calculated signal weights.

\section{Method}

Eight right-handed and healthy students (4 females; ages, 20-37 years, $M=25$ ) participated for course credit. Apparatus and stimuli were the same as those in Experiment 1. The design consisted of three within-participants variables: axis (radial or tangential), startpoint (ipsi- or contralateral), and standard stimulus (3/6, 4.5/4.5, or $6 / 3$ ). The participants explored with the index finger. Standard stimuli were compared with bumps with consistent force and position signals $(2.5-6.5 \mathrm{~mm}$; steps of $0.5 \mathrm{~mm} ;=9$ comparisons; 18 repetitions/pairing and condition). Single trials were conducted as in Experiment 1, except that the participants always stroked across two bumps (standard and comparison; located $+50 \mathrm{~mm}$ and $-50 \mathrm{~mm}$ from the center) and then indicated which of the two felt higher. The experiment consisted of three sessions ( $\sim 90 \mathrm{~min}$ each) including training and experimental blocks (6 repetitions/pairing and condition; random order). In the last session, there was a free exploration block (144 trials).

We determined individual psychometric functions for each standard and exploratory condition (including 162 trials each; details in Drewing \& Ernst, 2006). We fitted cumulative Gaussian functions to the psychometric functions, using the psignifit toolbox (Wichmann $\&$ Hill, 2001) and estimated PSEs by the Gaussian parameter $\mu$ and JNDs by $\sigma\left(=84 \%\right.$ discrimination thresholds). From PSEs $\left(A_{\mathrm{PSE}}\right)$ for the standards $3 / 6$ and $6 / 3\left(A_{P} / A_{F}\right)$, we calculated force signal weights $w_{F}^{e 2}$ :

$$
w_{F}^{e 2}=\frac{A_{\mathrm{PSE}}-A_{P}}{A_{F}-A_{P}} .
$$

We assumed that perceived amplitudes of the standard (ST) and a stimulus corresponding to the PSE are equal $\left(\hat{s}^{\mathrm{PSE}}=\hat{s}^{\mathrm{ST}}\right)$. Given that only force and position signals contribute to the percept and that signal estimates equal the signals, it follows from Equation 1 that

$$
\begin{aligned}
w_{F} A_{\mathrm{PSE}}+\left(1-w_{F}\right) A_{\mathrm{PSE}} & =w_{F} A_{F}+\left(1-w_{F}\right) A_{P} \\
& \rightarrow w_{F}=\frac{A_{\mathrm{PSE}}-A_{P}}{A_{F}-A_{P}}=w_{F}^{e 2} .
\end{aligned}
$$

That is, the weight $w_{F}^{e 2}$ is an estimate of the force signal estimate's weight $w_{F}$ in Equation 1. If the assumptions are violated, the interpretation of $w_{F}^{e 2}$ changes as described for $w_{F}^{e 1}$ (Equation 11).

\section{Results}

PSEs and weights. As should be the case, PSEs for the standard (Table 1) with consistent position and force signals $\left(A_{P} / A_{F}: 4.5 / 4.5\right)$ were, on average, close to the signals' actual values and did not differ between conditions ( $p$ s $>$ .05 in an ANOVA; variables: axis and startpoint). Force signal weights were lower for radial exploration starting contralateral to the hand, as compared with the three other directions (Figure 4B). Furthermore, they were lower for the $3 / 6$ stimulus than for the $6 / 3$ stimulus ( 0.59 vs. 0.40 ). This finding is at odds with previous reports (e.g., Experiment 1, here; Drewing \& Ernst, 2006). In an ANOVA (axis, startpoint, and standard stimulus), the main effect 
Table 1

Experiment 2: Average Points of Subjective Equality and Standard Errors Over the 8 Participants (in Millimeters)

\begin{tabular}{|c|c|c|c|c|c|c|c|c|}
\hline \multirow{3}{*}{$\begin{array}{c}\text { Standard Stimulus } \\
\text { (Position Signal/Force Signal) }\end{array}$} & \multicolumn{4}{|c|}{ Tangential Axis } & \multicolumn{4}{|c|}{ Radial Axis } \\
\hline & \multicolumn{2}{|c|}{$\begin{array}{l}\text { Ipsilateral } \\
\text { Startpoint }\end{array}$} & \multicolumn{2}{|c|}{$\begin{array}{l}\text { Contralateral } \\
\text { Startpoint }\end{array}$} & \multicolumn{2}{|c|}{$\begin{array}{l}\text { Ipsilateral } \\
\text { Startpoint }\end{array}$} & \multicolumn{2}{|c|}{$\begin{array}{c}\text { Contralatera } \\
\text { Startpoint }\end{array}$} \\
\hline & $M$ & $S E$ & $M$ & $S E$ & $M$ & $S E$ & $M$ & $S E$ \\
\hline $3.0 / 6.0$ & 4.83 & 0.11 & 4.60 & 0.13 & 4.77 & 0.10 & 4.89 & 0.04 \\
\hline $4.5 / 4.5$ & 4.62 & 0.05 & 4.58 & 0.05 & 4.67 & 0.06 & 4.55 & 0.05 \\
\hline $6.0 / 3.0$ & 4.69 & 0.10 & 4.95 & 0.12 & 4.72 & 0.09 & 4.79 & 0.08 \\
\hline
\end{tabular}

of standard stimulus $[F(1,7)=92.7, p<.001]$ and the startpoint $\times$ axis interaction $[F(1,7)=5.8, p<.05]$ were significant (other $p \mathrm{~s}>.05$ ).

JNDs. Amplitude discrimination was better for exploration along the tangential than along the radial axis
(Figure 4A). In an ANOVA (variables: axis, startpoint, and standard stimulus), only the main effect of axis was significant $[F(1,7)=6.9, p<.05]$.

Manipulation checks. Actual amplitudes deviated negligibly from the defined ones. Across 96 cases (ex-
A

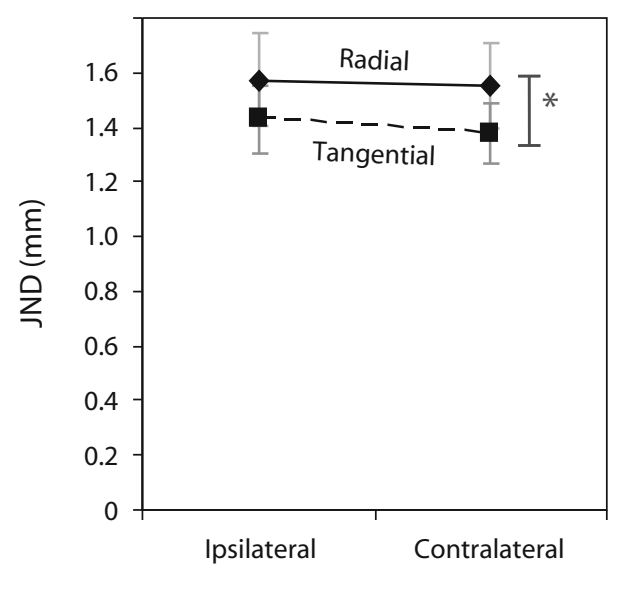

Startpoint
B

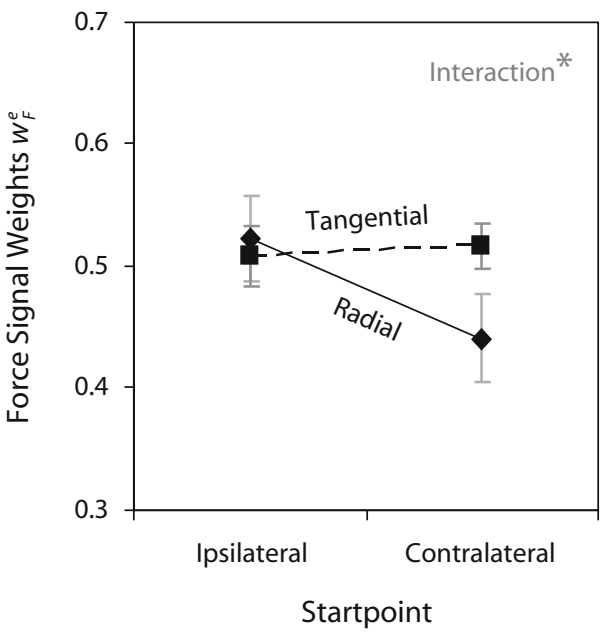

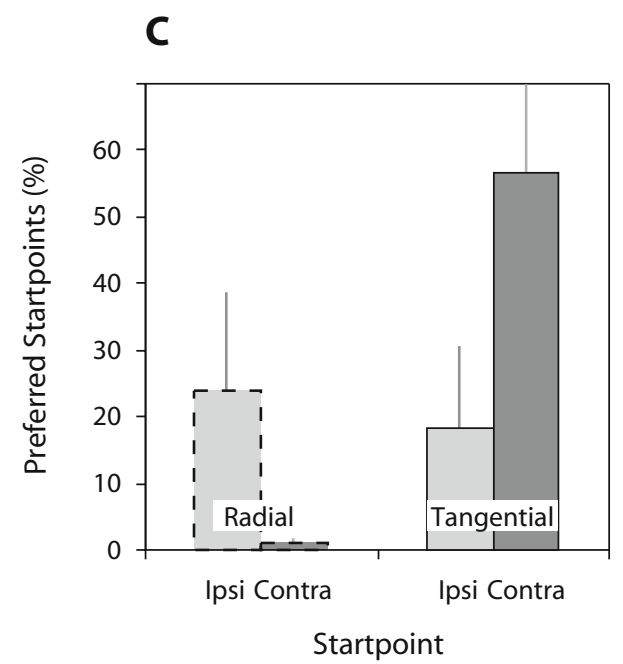

Figure 4. Experiment 2. (A) Just-noticeable differences (JNDs) and (B) force signal weights and standard errors (8 participants) as a function of axis and startpoint, collapsed across standard stimulus. (C) Percentages and standard errors of participants' choices of startpoint and axis in free exploration. 
ploratory conditions $\times$ stimuli $\times$ positions in stroke), mean amplitudes ranged from $99 \%$ to $106 \%$ of defined amplitudes. Standard deviations did not exceed $0.15 \mathrm{~mm}$. But again, exploration along the tangential axis was faster and less forceful than that along the radial axis (16.0 vs. $14.6 \mathrm{~cm} / \mathrm{sec}, 1.94$ vs. $2.03 \mathrm{~N}$ ). In ANOVAs (axis and startpoint), the effects of axis were significant [velocity, $F(1,7)=17.0, p<.001$; force, $F(1,7)=11.2, p<.02$; other $p \mathrm{~s}>.1]$.

Free exploration. Percentages of choices in the free exploration block indicated an overall preference for explorations along the tangential axis starting contralateral to the hand used (Figure 4C). In overall binomial tests, relative frequencies of choice differed from $50 \%$ for either pairing of two exploratory directions (all $p$ s $<$ $.01)$. Individual tests revealed that each participant significantly preferred one direction to all others (all $p \mathrm{~s}<$ .01 ; tangential-contra, 5 participants; tangential-ipsi, 1; radial-ipsi, 2).

\section{Discussion}

Experiment 2 measured the weights of force and position signals in perceived bump amplitude and the reliability of the amplitude percept. Measured reliability was lower (higher JND) for radial than for tangential directions, independent of startpoint. Force signal weight was lower for radial exploration with a contralateral startpoint than for tangential explorations. But for radial exploration with an ipsilateral startpoint, it was similar to tangential explorations. Originally, we had predicted from reliability-dependent weighting that the percept's reliability would covary with the force signal estimate weight. Measured reliabilities varied, but not in a one-to-one correspondence with the empirical weights. Importantly, the observed differences in reliability also do not correspond with our conclusion in Experiment 1 -namely, that estimate weights actually do not differ between exploratory directions (and effects in empirical weights are due to bias). Hence, reliability-dependent weighting cannot explain the effects of exploratory direction.

Can biases in estimating amplitude from force signals explain the present findings? From Experiment 1, we concluded that a factor $m_{F}$ relating force signal amplitudes $A_{F}$ and amplitude estimates $\hat{s}_{F}\left(\hat{s}_{F}=m_{F} A_{F}\right)$ is larger in tangential than in radial exploration. Such bias affects the interpretation of empirical measures. First, such bias affects the measurement of the percept's reliability by JNDs as follows. The percept's reliability refers to units of the brain's amplitude estimate $\hat{s}$ (cf. Equation 2) and might be thought of in terms of neuronal or "internal" variance in the responses to a single stimulus. In contrast, a JND is a physical amplitude difference, $\Delta A$, between two stimuli. It is that specific difference, $\Delta A$, that results in that specific (mean) difference, $\Delta \hat{s}$, between two amplitude estimates (distance between internal response distributions) that corresponds to internal variance. Importantly, this amplitude difference depends on the mapping of amplitudes $A$ on estimates $\hat{s}(\hat{s}=m A$; cf. above, differences between signals can be disregarded): With a larger mapping factor $m$, the same estimate difference, $\Delta \hat{s}$, results from a smaller physical difference, $\Delta A$ (because $\Delta \hat{s}=m \Delta A$ ). Then, the same internal variance (corresponding to a specific $\Delta \hat{s}$ ) results in a smaller JND $(=\Delta A)$. That means that a larger mapping factor $m$ predicts smaller JNDs without change in the percept's reliability. Here, this is predicted and observed for tangential, as compared with radial, exploration. Second, the interpretation of force signal weights, $w_{F}^{e}$, is affected by a mapping factor, $m_{F} \neq 1$, according to Equation 11B. Then, $w_{F}^{e}=m_{F} w_{F} /\left(m_{F} w_{F}+m_{P} w_{p}\right)$. One can see that a smaller factor, $m_{F}$, for radial, as compared with tangential, exploration alone predicts a smaller empirical weight, $w_{F}^{e}$. We observed a smaller weight in Experiment 2 for radial exploration with a contralateral, but not with an ipsilateral, startpoint. Taken together, biases in force signal estimates explain the present findings well, with the single exception of the weight in the radial condition with an ipsilateral startpoint.

Finally, we observed that exploratory preferences corresponded to directions with minimal JNDs-similar to what we had expected. JNDs were lower for tangential than for radial exploration, and the participants preferred tangential over radial exploration. Individual results corroborate the conclusion that participants recognize and prefer directions that allow them to keep judgment noise low: Within their individual range (minimal to maximal JNDs across directions), the participants preferred directions in which the JNDs were, on average, at only $17 \%$ above their minimum JND (i.e., at $83 \%$ below maximum).

\section{CONCLUSION}

We investigated how exploratory movement (variation of direction and finger used) influences the integration of force and position signals into haptically perceived bump amplitude. Under each exploratory condition, both signals contributed to the percept, and, as was predicted, their integration followed a weighted-averaging scheme. Effects of exploratory condition could, however, not be explained by reliability-dependent weighting - which is in contrast to our prediction. We rather conclude that exploratory condition affected the mapping of force signals on amplitude estimates, with the same force signal amplitudes resulting in larger estimates for tangential than for radial exploration and for index finger than for thumb exploration. Such biases explain observed exploratory effects: higher empirical force signal weights, larger amplitude percepts, and more reliable discrimination in the corresponding conditions. We suggest the inclusion of processes that derive signal-specific estimates from the single types of signals when extending models of signal integration to active touch. In addition, our results emphasize the active role of the explorer. Explorers utilized the exploratory movement that minimizes judgment noise.

Why were the amplitude estimates derived from the force signal larger for tangential than for radial exploration? We will first consider probable mechanisms underlying the sensing of force signals. The force signal, which acts laterally upon the finger, has been identified with acceleration and deceleration of the exploratory movement and with shear forces acting laterally on the skin 
(Hayward, 2008; Robles-De-La-Torre \& Hayward, 2001). That is, its sensing probably involves muscle receptor subsystems that signal rate of change in muscle length (which corresponds to velocity/acceleration) and cutaneous receptors that are particularly sensitive to shear force. It seems unlikely that the larger estimates for tangential than for radial exploration relate to cutaneous sensing via shear force: Shear forces are perceived more accurately when the skin is stretched longitudinally, as compared with transversely (Keyson \& Houtsma, 1995). In contrast, here, the finger crossed the bump transversely with tangential exploration (from left to right side, or vice versa), and longitudinally with radial exploration. Hence, we rather believe that the biases relate to the sensing of force signal effects involving the entire finger. Consider how the finger is anatomically connected to the hand: The mere mechanical effect of lateral forces on the entire finger is probably more pronounced for force pulses from the left or right than for pulses from ahead or behind the fingertip (= radial exploration). These mechanical differences might have magnified the sensations arising from the force signal in tangential exploration.

But why did we observe differential mapping of force signals on estimates, rather than differential estimate reliability? We suggest that the brain's processing of the force signal does not take into account the entire pattern of lateral force change over time and space (which would allow the recovery of the detailed shape) but, rather, takes into account only reduced aspects of the signal, such as force amplitude or maximum force difference. A number of haptic illusions also suggest that the interpretation of complicated force patterns can be erroneous. For example, if people move one arm against a constant force, they can estimate the first arm's position appropriately using the other arm, but if the force varies during movement, they make systematic mistakes (Rymer \& D'Almeida, 1980). This also occurs with actively induced force changes (Roland, 1978). Still, force signals seem to play an important role for haptic perception of quite different properties, such as softness, friction, texture, length, or shape (Wydoodt, Gentaz, \& Streri, 2006).

\section{AUTHOR NOTE}

This research was supported by the Deutsche Forschungsgemeinschaft (DR 730/1-1, FOR 560). Thanks to Bianca Jovanovic, Florian Bayer, Christoph Rasche, Vincent Hayward, and two anonymous reviewers for many helpful suggestions, and to Michaela Demandt and Debora Peine for data collection. Correspondence concerning this article should be addressed to K. Drewing, Institute for Psychology, Giessen University, Otto-Behaghel-Str. 10F, 35394 Giessen, Germany (e-mail: knut .drewing@psychol.uni-giessen.de).

\section{REFERENCES}

Alais, D., \& BURR, D. (2004). The ventriloquist effect results from nearoptimal bimodal integration. Current Biology, 14, 257-262.

Backus, B. T., Banks, M. S., van Ee, R., \& Crowell, J. A. (1999). Horizontal and vertical disparity, eye position, and stereoscopic slant perception. Vision Research, 39, 1143-1170.
Drewing, K., \& ERnst, M. O. (2006). Integration of force and position cues for shape perception through active touch. Brain Research, 1078, $92-100$.

Drewing, K., WiECKI, T. V., \& ERNST, M. O. (2008). Material properties determine how force and position signals combine in haptic shape perception. Acta Psychologica, 128, 264-273.

ERNST, M. O., \& BANKS, M. S. (2002). Humans integrate visual and haptic information in a statistically optimal fashion. Nature, 415, 429-433.

GeIsSer, S., \& Greenhouse, S. W. (1958). An extension of Box's results on the use of the $F$-distribution in multivariate analysis. Annals of Mathematical Statistics, 29, 885-891.

Gepshtein, S., Burge, J., Ernst, M. O., \& Banks, M. S. (2005). The combination of vision and touch depends on spatial proximity. Journal of Vision, 5, 1013-1023.

Gibson, J. J. (1962). Observations on active touch. Psychological Review, 69, 477-491

HAYWARD, V. (2008). Haptic shape cues, invariants, priors, and interface design. In M. Grunwald (Ed.), Human haptic perception: Basics and applications (pp. 381-392). Basel: Birkhäuser.

Hillis, J. M., Watt, S. J., Landy, M. S., \& BanKs, M. S. (2004). Slant from texture and disparity cues: Optimal cue combination. Journal of Vision, 4, 967-992.

JACOBS, R. A. (2002). What determines visual cue reliability? Trends in Cognitive Sciences, 6, 345-350.

KAIM, L., \& DREWING, K. (2007). Influence of parametric variation in exploratory movement on signal integration for haptic shape perception. In H. H. Bülthoff, A. Chatziastros, H. A. Mallot, \& R. D. Ulrich (Eds.), 10th Tübinger Perception Conference (p. 99). Kirchentellinsfurt: Knirsch

Keyson, D. V., \& Houtsma, A. J. M. (1995). Directional sensitivity to a tactile point stimulus moving across the fingerpad. Perception \& Psychophysics, 57, 738-744.

Landy, M. S., Maloney, L. T., Johnston, E. B., \& Young, M. (1995). Measurement and modeling of depth cue combination: In defense of weak fusion. Vision Research, 35, 389-412.

LEDERMAN, S. J., \& KLATZKY, R. L. (1987). Hand movements: A window into haptic object recognition. Cognitive Psychology, 19, 342-368.

Perotti, V. J., Todd, J. T., Lappin, J. S., \& Phillips, F. (1998). The perception of surface curvature from optical motion. Perception \& Psychophysics, 60, 377-388.

Robles-De-La-Torre, G., \& Hayward, V. (2001). Force can overcome object geometry in the perception of shape through active touch. $\mathrm{Na}$ ture, 412, 445-448.

RoLAND, P. E. (1978). Sensory feedback to the cerebral cortex during voluntary movement in man. Behavioral \& Brain Sciences, 1, 129-171.

Rosas, P., Wagemans, J., Ernst, M. O., \& Wichmann, F. A. (2005). Texture and haptic cues in slant discrimination: Reliability-based cue weighting without statistically optimal cue combination. Journal of the Optical Society of America A, 22, 801-809.

Rymer, W. Z., \& D'AlmeidA, A. (1980). Joint position sense: The effects of muscle contraction. Brain, 103, 1-22.

Srinivasan, M. A., \& LaMotTe, R. H. (1995). Tactual discrimination of softness. Journal of Neurophysiology, 73, 88-101.

Voisin, J., LamarRe, Y., \& Chapman, C. E. (2002). Haptic discrimination of object shape in humans: Contribution of cutaneous and proprioceptive inputs. Experimental Brain Research, 145, 251-260.

WichmanN, F. A., \& HiLl, N. J. (2001). The psychometric function: I. Fitting, sampling, and goodness of fit. Perception \& Psychophysics, 63, 1293-1313.

Wydoodt, P., Gentaz, E., \& Streri, A. (2006). Role of force cues in the haptic estimations of a virtual length. Experimental Brain Research, 171, 481-489.

Young, M. J., Landy, M. S., \& Maloney, L. T. (1993). A perturbation analysis of depth perception from combinations of texture and motion cues. Vision Research, 33, 2685-2696.

(Manuscript received August 7, 2008; revision accepted for publication January 31, 2009.) 